Pacific Journal of Mathematics

TOPOLOGICAL ENTROPY AND RECURRENCE OF

D 


\title{
TOPOLOGICAL ENTROPY AND RECURRENCE OF COUNTABLE CHAINS
}

\author{
IBRAHIM A. SALAMA
}

\begin{abstract}
We consider a symbolic dynamical system $(X, \sigma)$ on a countable state space. We introduce a kind of topological entropy for such systems, denoted $h^{*}$, which coincides with usual topological entropy when $X$ is compact. We use a pictorial approach, to classify a graph $\Gamma$ (or a chain) as transient, null recurrent, or positive recurrent. We show that given $0 \leq \alpha \leq \beta \leq \infty$, there is a chain whose $h^{*}$ entropy is $\beta$ and where Gurevic entropy is $\alpha$. We compute the topological entropies of some classes of chains, including larger chains built up from smaller ones by a new operation which we call the Cartesian sum.
\end{abstract}

Introduction. The importance of subshifts of finite type in ergodic theory and dynamical systems is well known. One needs also to study chains on a countably infinite set in order to analyze problems in various fields such as differentiable dynamics, coding for magnetic recording, nonuniqueness of equilibrium states in statistical mechanics, formal languages and automata, or even to analyze arbitrary subshifts. (See [3], [9], [6], [2], [1], [10], respectively.)

Let $\Gamma$ be a strongly connected directed graph on a countable set of vertices $S=\left\{s_{1}, s_{2}, \ldots\right\}$, and let

$$
X(\Gamma)=\left\{x \in S^{z} \mid \text { for all } i \text {, there is an edge in } \Gamma \text { from } x_{i} \text { to } x_{i+1}\right\} .
$$

If $S$ has the discrete topology and $S^{z}$ the product topology, then in the induced topology $X(\Gamma)$ (or simply $X$ ), together with the shift transformation $\sigma$ defined by $(\sigma x)_{i}=x_{i+1}$ for all $i$, is a (non-compact) dynamical system, called the chain determined by the directed graph $\Gamma$. The topological entropy of $X$ may be determined using Bowen's definition, to obtain $h_{B}(X)$ (see [8] for a definition). This definition depends on the metric we put on $X$. We consider the following two metric spaces.

1. For $x, y \in X$ define

$$
d_{1}(x, y)=\sum_{i} \frac{1-\delta\left(x_{i}, y_{i}\right)}{2^{|i|}},
$$

where $\delta(s, t)=1$ if $s=t$ and 0 if $s \neq t$. 
2. Let $S=\{1,2, \ldots$,$\} and define$

$$
d_{2}(x, y)=\sum_{i} \frac{1}{2^{|i|}} \frac{1-\delta\left(x_{i}, y_{i}\right)}{\min \left(x_{i}, y_{i}\right)}
$$

(we may use $S=\{1 / n, n \geq 1\}$ and define $d_{2}(x, y)=\sum_{i}\left|x_{i}-y_{i}\right| / 2^{|i|}$ ). On the other hand and without reference to any metric, we may use Gurevic's definition (Gurevic, [4]), $h_{G}(X)=h_{G}(\Gamma)=\sup _{\Gamma^{\prime}<\Gamma} h\left(\Gamma^{\prime}\right)$, where the sup is taken over all (connected) finite subgraphs $\Gamma^{\prime}$ of $\Gamma$ (and $h\left(\Gamma^{\prime}\right)$ is the usual topological entropy of the subshift of finite type determined by $\Gamma^{\prime}$, the logarithm of the maximal eigenvalue of the transition matrix).

Another possible approach, suggested by Gurevic [4], is to let $\bar{S}$ be the one-point compactification of $S$, and $\bar{X}$ the closure of $X$ in $\bar{S}^{z}$. Then $\bar{X}$ is compact, and we may define $h_{c}(X)$ to be the ordinary topological entropy of $(\bar{X}, \sigma)$.

Fix a vertex $S$ in $\Gamma$ and define

$B_{s}^{(n)}=$ number of paths of length $n$ in $\Gamma$ from $s$ to $s$;

$f_{s}^{(n)}=$ number of paths of length $n$ in $\Gamma$ from $s$ to $s$ with no other occurrences of $s$ in between;

$$
\begin{aligned}
& F(\Gamma, s, z)=\sum f_{s}^{(n)} z^{n} \\
& B(\Gamma, s, z)=\sum B_{s}^{(n)} z^{n} \\
& F^{\prime}(\Gamma, s, z)=\sum n f_{s}^{(n)} z^{n-1} .
\end{aligned}
$$

Let $L_{\Gamma}$ and $R_{\Gamma}$ be the radii of convergence of $F(\Gamma, s, z)$ and $B(\Gamma, s, z)$, respectively (they are independent of $s$ ). We will abbreviate $L_{\Gamma}$ by $L$ and $R_{\Gamma}$ by $R$ (generally, $L_{\Gamma_{i}}$ by $L_{i}$ and $R_{\Gamma_{i}}$ by $R_{i}$ ).

Vere-Jones ([11], [12]) studied the classification of the graph $\Gamma$ as transient, null recurrent, or positive recurrent according to the following table:

$$
\begin{array}{cccc} 
& \text { null } & \text { positive } \\
F(\Gamma, s, R) & <1 & =1 & =1 \\
F^{\prime}(\Gamma, s, R) & =\infty & =\infty & <\infty \\
B(\Gamma, s, R) & <\infty & =\infty & =\infty \\
\lim _{n \rightarrow \infty} B_{s}^{(n)} R^{n} & =0 & =0 & >0
\end{array}
$$

Gurevic ([4], [5]) showed that if $X$ is a connected chain with the $d_{2}$-metric, then $h_{G}(X)=h_{c}(X)$; and if $\Gamma$ is a connected graph with $h_{G}(\Gamma)<\infty$, then $h_{G}(\Gamma)=-\log R$.

The paper is organized as follows: In $\S 1$ we introduce a new definition for the topological entropy of a symbolic dynamical system 
$(X, \sigma)$, which we call $h^{*}$. For a locally finite chain $X$, we show that $h_{B}(X)=h^{*}(X)$ when the $d_{1}$-metric is used; and that $h_{B}(X)=h_{G}(X)$ when the $d_{2}$-metric is used. In $\S 2$ we consider a geometric or pictorial approach to classify chains as transient, null recurrent, or positive recurrent. In $\S 3$ we show that given $1<\alpha \leq \beta$, there exists a recurrent uniformly locally finite graph $\Gamma$ such that $h_{G}(\Gamma)=\log \alpha$ and $h^{*}(\Gamma)=\log \beta$. If $\alpha=\beta$, then the $\Gamma$ that we construct is positive recurrent. In $\S 4$ we consider some computation examples.

1. The $h^{*}$ entropy. Let $(X, \sigma)$ be a symbolic dynamical system with countable state space $S=\left\{s_{1}, s_{2}, \ldots\right\}$. If $S$ is finite, then $X$ is compact and the topological entropy of $X$ is given by $h(X)=\lim _{n} \frac{1}{n} \log B^{(n)}$, where $B^{(n)}$ is the number of blocks of length $n$ in $X$. Taking this formula and applying it "formally" to symbolic dynamical systems with infinite state space $S$, we will end up assigning the same value, namely $\infty$, to these systems. However, it is reasonable to consider the rate of growth of the number of blocks in $X$ starting with a fixed symbol or generally starting with a fixed block. Based on this notion we introduce a new definition for the topological entropy of $(X, \sigma)$, which we shall call $h^{*}$, formulated as follows.

Definition Let $T_{s}^{(n)}$ be the number of blocks of length $n$ in $X$ starting with $s \in S$. The entropy of $X$ relative to $s$ is defined by

$$
h^{*}(X \mid s)=\varlimsup_{n} \frac{1}{n} \log T_{s}^{(n)},
$$

and the entropy of $X$ is defined by

$$
h^{*}(X)=\sup _{s \in S} h^{*}(X \mid s) .
$$

We observe that if $X$ is a finite connected chain, then $h^{*}(X)=$ $h(X)=h_{G}(X)=h_{B}(X)$. Also, it is easy to see that if $X$ is transitive, then $h^{*}(X)=h^{*}(X \mid s)$ for all $s \in S$, and if $X$ is a countable connected chain, then $h_{G}(X) \leq h^{*}(X)$.

A chain $X$ is locally finite if the corresponding graph $\Gamma$ satisfies: If $s$ is a state of $\Gamma$ then the number of arrows coming in and going out of $s$ is finite. The chain is uniformly locally finite if the number of arrows coming in and going out of any state is less than some fixed number, say $m$.

THEOREM (1.1). If $X$ is a locally finite connected chain with the metric $d_{1}$, then $h^{*}(X)=h_{B}(X)$. 
Proof. Let $K$ be a compact subset of $X$. Then for every $i$, the number of different symbols appearing in the $i$ th place in $K$ is finite. For $\varepsilon=2^{-i}, r_{n}(\varepsilon, K)$ is the number of different blocks in $K$ of the form $s_{-i} \cdots s_{n+i}$. Let $N_{-i}$ be the set of symbols appearing in $K$ in the $-i$ th place. Then $r_{n}(\varepsilon, K)=\sum_{s \in N_{-}} r_{n}^{s}(\varepsilon, K)$, where $r_{n}^{s}(\varepsilon, K)$ is the number of blocks in $K$ of the form $s s_{-(i-1)} \cdots s_{n+i}$ and $s \in N_{-i}$. Since $r_{n}(\varepsilon, K)$ is the sum of $\#\left\{N_{-i}\right\}$ nonnegative sequences, there is $s_{0} \in N_{-i}$ such that

$$
r(\varepsilon, K)=\varlimsup \frac{\lim }{\frac{1}{n}} \log r_{n}(\varepsilon, K)=\varlimsup \frac{\lim }{n} \log r_{n}^{S_{0}}(\varepsilon, K) \leq h^{*}(X)
$$

Hence $h_{B}(X) \leq h^{*}(X)$.

Now, since $X$ is locally finite, for $s \in S, K_{s}=\left\{x \in X \mid x_{0}=s\right\}$ is compact. If $\varepsilon=2^{-i}$, then $r_{n}\left(\varepsilon, T^{-i} K_{s}\right)$ is the number of blocks in $K_{s}$ of the form $s s_{-(i-1)} \cdots s_{n+1}$, and $h^{*}(X \mid s)=\varlimsup \lim \frac{1}{n} \log r_{n}\left(\varepsilon, T^{-i} K_{s}\right) \leq$ $h_{B}(X)$. Thus, $h^{*}(X) \leq h_{B}(X)$.

The notion of the $h^{*}$-entropy may be extended to a general topological space (not necessarily compact) and a homeomorphism $T: X \rightarrow X$ as follows. For an open cover $\alpha$ of $X$ and a compact $K \subset X$ let $N_{K}^{n}(\alpha)$ denote the minimum number of sets in $\bigvee_{i=0}^{n-1} T_{\alpha}^{-i}$ needed to cover $K$, and let $H_{K}^{n}(\alpha)=\log N_{K}^{n}(\alpha)$. The entropy of $T$ relative to $\alpha$ given compact $K \subset X$ is defined as $h^{*}(T, \alpha ; K)=\varlimsup_{\lim _{n}} \frac{1}{n} H_{K}^{n}(\alpha)$. The entropy of $T$ relative to $\alpha$ is defined as $h^{*}(T, \alpha)=\sup _{K \subset X} h^{*}(T, \alpha ; K)$, where $K$ is compact. Finally, the entropy of $T$ is defined as $h^{*}(T)=\sup _{\alpha} h^{*}(T, \alpha)$. The following fact follows from a straightforward argument.

Proposition (1.2). If $X$ is compact, then $h^{*}(T)=h(T)$.

Proposition (1.3). If $X$ is a connected chain with the $d_{2}$-metric, then $h_{B}(X)=h_{G}(X)$.

Proof. Let $K$ be a compact subset of $X, r_{n}(\varepsilon, K)=$ the smallest cardinality of an $(n, \varepsilon)$-spanning set of $K, r(\varepsilon, K)=\varlimsup_{n} \frac{1}{n} \log r_{n}(\varepsilon, K)$, and $h_{B}(K)=\lim _{\varepsilon \rightarrow 0} r(\varepsilon, K)$, so that $h_{B}(X)=\sup _{K \subset X} h_{B}(K)$.

If $X^{\prime}$ is a connected finite subchain of $X$, then $h_{G}\left(X^{\prime}\right) \leq h_{B}(X)$; hence $h_{G}(X) \leq h_{B}(X)$. On the other hand, let $\bar{X}$ be the completion of $X$ in the $d_{2}$-metric. Since $\left(X, d_{2}\right)$ is totally bounded, $\left(\bar{X}, d_{2}\right)$ is compact. If $X^{\prime \prime}$ is a compact subset of $X$, then $X^{\prime \prime}$ is compact as a subset of $\bar{X}$, and $h_{B}\left(X^{\prime \prime}\right) \leq h_{B}(\bar{X})=h(\bar{X})=h_{G}(X)$. Hence $h_{B}(X) \leq h_{G}(X)$. 
2. Classification of chains. In this section we present a criterion enabling us to decide if a connected graph $\Gamma$ is transient, null recurrent, or positive recurrent. Our approach is geometric or pictorial in nature, avoiding the computational and combinatorial problems usually encountered. The idea is to relate the value of $h_{G}(\Gamma)$ to the values of $h_{G}\left(\Gamma^{\prime}\right)$, where $\Gamma^{\prime}$ is either a subgraph of $\Gamma$ or a graph containing $\Gamma$. In general terms, our results on this question may be summarized as follows: $\Gamma$ is transient if and only if we can "expand" or "contract" $\Gamma$ without changing $h_{G}, \Gamma$ is positive-recurrent if and only if any "expansion" or "contraction" of $\Gamma$ will change $h_{G}$ in the right direction; and $\Gamma$ is null-recurrent if and only if we can "contract" $\Gamma$ without changing $h_{G}$ but any "expansion" of $\Gamma$ will increase $h_{G}$. We prove the following.

Theorem (2.1). (i) If $\Gamma_{0}<\Gamma_{2}$ and $h_{G}\left(\Gamma_{0}\right)=h_{G}\left(\Gamma_{2}\right)$, then $\Gamma_{0}$ is transient.

(ii) If $\Gamma_{0}$ is transient, then there exists $\Gamma_{2}>\Gamma_{0}$ such that $h_{G}\left(\Gamma_{0}\right)=$ $h_{G}\left(\Gamma_{2}\right)$ and $\Gamma_{2}$ is transient.

THEOREM (2.2). Let $\Gamma_{0}$ be such that $R_{0}=L_{0}$. Then there exists $\Gamma<\Gamma_{0}$ such that $h_{G}(\Gamma)=h_{G}\left(\Gamma_{0}\right)$.

THEOREM (2.3). The following conditions for $\Gamma_{0}$ are equivalent:

(i) $\Gamma_{0}$ is positive recurrent.

(ii) $R_{0}<L_{0}$.

(iii) For all $\Gamma_{1}<\Gamma_{0}, h_{G}\left(\Gamma_{1}\right)<h_{G}\left(\Gamma_{0}\right)$.

(iv) $F\left(\Gamma_{0}, s, L_{0}\right)>1$.

COROLlary (2.4). If $\Gamma_{0}$ is transient, then there exists $\Gamma_{1}<\Gamma_{0}$ such that $h_{G}\left(\Gamma_{1}\right)=h_{G}\left(\Gamma_{0}\right)$ and $\Gamma_{1}$ is transient.

CoRollaRy (2.5). $\Gamma_{0}$ is recurrent if and only if for all $\Gamma_{2}>\Gamma_{0}$, $h_{G}\left(\Gamma_{2}\right)>h_{G}\left(\Gamma_{0}\right)$.

COROLLARY (2.6). $\Gamma_{0}$ is null-recurrent if and only if: (i) there exists $\Gamma_{1}<\Gamma_{0}$ such that $h_{G}\left(\Gamma_{1}\right)=h_{G}\left(\Gamma_{0}\right)$, and (ii) for all $\Gamma_{2}>\Gamma_{0}$ we have $h_{G}\left(\Gamma_{2}\right)>h_{G}\left(\Gamma_{0}\right)$.

Proof of Theorem (2.1). (i) Let $s$ be a state in $\Gamma_{0}$ and $\Gamma_{2}$. Then $F\left(\Gamma_{2}, s, R_{2}\right) \leq 1$. Since $\Gamma_{0}<\Gamma_{2}$ and $R_{0}=R_{2}$, we have $F\left(\Gamma_{0}, s, R_{0}\right)<1$.

(ii) Since $\Gamma_{0}$ is transient, $F\left(\Gamma_{0}, s, R_{0}\right)=\sum_{n} f_{s}^{(n)} R_{0}^{n}<1$. Hence, for some $m$ we can find a positive integer $k$ such that $k>f_{s}^{(m)}$ 
and $\sum_{n \neq m} f_{s}^{(n)} R_{0}^{n}+k R_{0}^{m}<1$. Let $\Gamma_{2}$ be the graph obtained by adding a loop of length $\left(k-f_{s}^{(m)}\right)$ based at $s$ to $\Gamma_{0}$. Thus $\Gamma_{0}<\Gamma_{2}$ and $F\left(\Gamma_{2}, s, R_{0}\right)<1$. Since $R_{2} \leq R_{0}$, then $F\left(\Gamma_{2}, s, R_{2}\right) \leq F\left(\Gamma_{2}, s, R_{0}\right)<1$, and $\Gamma_{2}$ is transient. To show $R_{0}=R_{2}$, note that $L_{0}=L_{2}$ and $R_{2} \leq R_{0} \leq L_{0}$. If $R_{2}<R_{0}$, then $R_{2}<L_{2}$ and $F^{\prime}\left(\Gamma_{2}, s, R_{2}\right)<\infty$, contradicting that $\Gamma_{2}$ is transient.

Proof of Theorem (2.2). For a state $s$ in $\Gamma_{0}$, let $\operatorname{Fol}(s)=\left\{s^{\prime} \mid\right.$ there is an arrow from $s$ to $s^{\prime}$ in $\left.\Gamma_{0}\right\}$. Let $s_{0}$ be a state in $\Gamma_{0}$ such that $\#$ Fol $\left(s_{0}\right) \geq 2$. Let $s^{*} \in \operatorname{Fol}\left(s_{0}\right)$, let $\Gamma_{1}$ be the subgraph obtained by removing the edge $s_{0} s^{*}$ from $\Gamma_{0}$, and let $\Gamma_{2}$ be the subgraph obtained by the removal from $\Gamma_{0}$ the edges $s_{0} s^{\prime}, s^{\prime} \in \operatorname{Fol}\left(s_{0}\right)-s^{*}$. Let $i$ be such that $L_{i}=\min \left(L_{1}, L_{2}\right)$. Then $R_{0} \leq R_{i} \leq L_{i}=L_{0}$. Since $L_{0}=R_{0}$, we have $R_{0}=R_{i}$ and $h_{G}\left(\Gamma_{0}\right)=h_{G}\left(\Gamma_{i}\right)$.

Proof of Theorem (2.3). (i) If $R_{0}<L_{0}$, then $F^{\prime}\left(\Gamma_{0}, s, R_{0}\right)<\infty$ and $\Gamma_{0}$ is positive recurrent. If $R_{0}=L_{0}$, then by Theorem (2.2) there is $\Gamma_{1}<\Gamma_{0}$ such that $R_{1}=R_{0}$. Hence by (i) of Theorem (2.1) $\Gamma_{1}$ is transient. Since $F^{\prime}\left(\Gamma_{1}, s, R_{0}\right)=\infty$ and $F^{\prime}\left(\Gamma_{1}, s, R_{0}\right) \leq F^{\prime}\left(\Gamma_{0}, s, R_{0}\right), \Gamma_{0}$ cannot be positive recurrent.

(ii) We show that: $\Gamma_{0}$ is not positive-recurrent if and only if there is $\Gamma_{1}<\Gamma_{0}, h_{G}\left(\Gamma_{1}\right)=h_{G}\left(\Gamma_{0}\right)$. So, assume that $\Gamma_{0}$ is not positiverecurrent, then by (i) above we have $R_{0}=L_{0}$; hence, by Theorem (2.2) there is $\Gamma_{1}<\Gamma_{0}$ such that $h_{G}\left(\Gamma_{1}\right)=h_{G}\left(\Gamma_{0}\right)$. On the other hand, if there exists $\Gamma_{1}<\Gamma_{0}$ with $h_{G}\left(\Gamma_{1}\right)=h_{G}\left(\Gamma_{0}\right)$, then $\Gamma_{0}$ is not positiverecurrent as shown in the proof of (i) above.

(iii) Assume $F\left(\Gamma_{0}, s, L_{0}\right)>1$. Since $F\left(\Gamma_{0}, s, R_{0}\right) \leq 1$, we have $R_{0}<$ $L_{0}$ and by (i) above $\Gamma_{0}$ is positive-recurrent. If $\Gamma_{0}$ is positive-recurrent, then $R_{0}<L_{0}$. Since $F\left(\Gamma_{0}, s, R_{0}\right)=1$, we have $F\left(\Gamma_{0}, s, L_{0}\right)>1$.

Finally, Corollary (2.4) follows from part (ii) of the proof of Theorem (2.3), Corollary (2.5) follows from Theorem (2.1), and Corollary (2.6) follows from part (ii) of the proof of Theorem (2.3) and Corollary (2.5).

3. Relation between $h_{G}$ and $h^{*}$. In this section we study the relation between $h_{G}$ and $h^{*}$ using the following class of countable graphs:

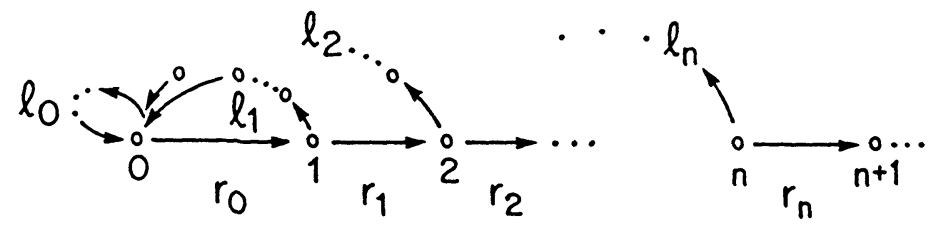


Here: $r_{i}=$ the number of paths from state $i$ to state $i+1$, and $l_{i}=$ the number of arcs between state $i$ and state 0 (as shown above). A graph of this form is indexed by two nonnegative integer sequences $\left\{r_{n}\right\},\left\{l_{n}\right\}$ and is denoted by $\Gamma\left(\left\{r_{n}\right\},\left\{l_{n}\right\}\right)$.

Let $\Gamma_{0}$ be any connected countable graph (not necessarily of the form above) and let $s$ be a state of $\Gamma_{0}$. Define $C_{s}^{(n)}=$ the number of sequences $s s_{i_{1}} \cdots s_{i_{n-1}}$ in $\Gamma_{0}$ such that $s_{j} \neq s$ for $j=i_{1}, \ldots, i_{n-1}$, and recall that $T_{s}^{(n)}=$ the number of sequences of length $n$ in $\Gamma_{0}$ that start with $s$. Let $C\left(\Gamma_{0}, s, z\right)=\sum_{n} C_{s}^{(n)} z^{n}, T\left(\Gamma_{0}, s, z\right)=\sum_{n} T_{s}^{(n)} z^{n}$, and $Q_{0}\left(A_{0}\right)$ be the radius of convergence of $C\left(\Gamma_{0}, s, z\right)\left(T\left(\Gamma_{0}, S, z\right)\right)$.

Towards proving the main result of this section, we use the following Lemmas:

LeMma (3.1). For a connected graph $\Gamma_{0}$, we have

$$
h^{*}\left(\Gamma_{0}\right)=\max \left\{\log Q_{0}^{-1}, h_{G}\left(\Gamma_{0}\right)\right\} \text {. }
$$

Proof. Let $s$ be a state in $\Gamma_{0}$. Then $T_{s}^{(n)}=\sum_{i=1}^{n} B_{s}^{(n-i)} C_{s}^{(i)}$, and $A_{0}=\min \left(R_{0}, Q_{0}\right)$. Since $h^{*}\left(\Gamma_{0}\right)=\log A_{0}^{-1}, h_{G}\left(\Gamma_{0}\right)=\log R_{0}^{-1}$, the result follows.

LEMMA (3.2). Let $\Gamma_{0}\left(\left\{r_{n}\right\},\left\{l_{n}\right\}\right)$ be a graph with associated sequence $\left\{C_{0}^{(n)}\right\}$. Then

(i) If $\lim _{n} \frac{1}{n} \log \prod_{i=1}^{n} r_{i}=\log \beta$, then $\lim _{n} \frac{1}{n} \log C_{0}^{(n)}=\log \beta$.

(ii) $h^{*}\left(\Gamma_{0}\right)=\max \left\{\log \beta, h_{G}\left(\Gamma_{0}\right)\right\}$.

Proof. Let $n_{i}=l_{i}+(i+1), i=0,1,2, \ldots$, and $W_{k}=\prod_{i=1}^{k} r_{i}, i=$ $1,2, \ldots\left(W_{0}=1\right)$. To count the number of sequences of length $k$ in $\Gamma_{0}$ that start with 0 , let $S_{k}=\left\{i=0,1, \ldots, k-1 \mid n_{i} \geq k\right\}$, then $C_{0}^{(k)}=\sum_{i \in S_{k}} W_{i}$ and $T_{0}^{(n)}=\sum_{i=1}^{n} B_{0}^{(n-i)} C_{0}^{(i)}$. Noting that $W_{k} \leq$ $C_{0}^{(k)} \leq \sum_{i=1}^{k} W_{i}$, and $\lim _{n} \frac{1}{n} \log \prod_{i=1}^{n} r_{i}=\lim _{n} \frac{1}{n} \log W_{n}=\log \beta$, then $\lim _{n} \frac{1}{n} \log C_{0}^{(n)}=\log \beta$. That shows (i) and (ii) follows by Lemma (3.1).

Lemma (3.3). Let $m<x<(m+1)$. Then there is a sequence $\left\{r_{n}\right\}$ such that

(i) $r_{n}=m$ or $m+1$,

(ii) for all $n$ :

$$
\prod_{i=1}^{n} r_{i} \leq x^{n}<\left(\frac{m+1}{m}\right) \prod_{i=1}^{n} r_{i}
$$


and

(iii) $\lim _{n} \frac{1}{n} \log \prod_{i=1}^{n} r_{i}=\log x$.

For a graph $\Gamma_{0}\left(\left\{r_{n}\right\},\left\{l_{n}\right\}\right)$, let $W_{n}=\prod_{i=1}^{n} r_{i}$, and let $W_{0}$ be the radius of convergence of the series $\sum_{n} W_{n} z^{n}$. Then by Lemma (3.2) we have

Lemma (3.4). For $\Gamma_{0}\left(\left\{r_{n}\right\},\left\{l_{n}\right\}\right)$, if $L_{0}=W_{0}$, then $h_{G}\left(\Gamma_{0}\right)=h^{*}\left(\Gamma_{0}\right)$.

Lemma (3.5). For $\Gamma_{0}\left(\left\{r_{n}\right\},\left\{l_{n}\right\}\right)$, if $\lim _{n} \frac{1}{n} \log \prod_{i=1}^{n} r_{i}=\log W_{0}^{-1}$ and $\left\{l_{n}\right\}$ is bounded (say by $k$ ), then $L_{0}=W_{0}$ and $h_{G}\left(\Gamma_{0}\right)=h^{*}\left(\Gamma_{0}\right)$.

Proof. Note that $f_{0}^{(n)} \leq \sum_{i=0}^{n} W_{i}$, hence $W_{0} \leq L_{0}$. To show that $L_{0} \leq W_{0}$, consider the sequence $\left\{n_{j} \mid l_{n} \neq 0\right\}$. Then for every $j$ we have $f_{0}^{\left(n_{j}+l_{n_{\jmath}}\right)} \geq W_{n_{j}}$, hence

$$
\varlimsup_{j} \frac{1}{n_{j}} \log f_{0}^{\left(n_{j}+l_{n_{j}}\right)} \geq \frac{\lim }{j} \frac{1}{n_{j}} \log W_{n_{j}}=\log W_{0}^{-1},
$$

and $L_{0} \leq W_{0}$. By Lemma (3.4) we have $h_{G}\left(\Gamma_{0}\right)=h^{*}\left(\Gamma_{0}\right)$.

Lemma (3.6). Let $\Gamma_{0}\left(\left\{r_{n}\right\},\left\{l_{n}\right\}\right)$ be such that $\left\{r_{n}\right\}$ is a special sequence satisfying the conditions of Lemma (3.3) and $\left\{l_{n}\right\}$ is bounded (say by $k$ ). Then $h_{G}\left(\Gamma_{0}\right)=h^{*}\left(\Gamma_{0}\right)$ and $\Gamma_{0}$ is positive recurrent.

Proof. As before consider the sequence $\left\{n_{j} \mid l_{n_{j}} \neq 0\right\}$. Then for every $j$ we have

$$
W_{n_{\jmath}}=\prod_{i=1}^{n_{\jmath}} r_{i} \leq f_{0}^{\left(n_{j}+l_{n_{j}}\right)} \quad \text { and } \quad \frac{W_{n_{j}}}{x^{n_{\jmath}+l_{n_{j}}}} \leq \frac{f_{0}^{\left(n_{\jmath}+l_{n_{j}}\right)}}{x^{n_{\jmath}+l_{n_{j}}}} .
$$

By (ii) of Lemma (3.3), we have $W_{n_{\jmath}} / x^{n_{\jmath}}>m /(m+1)>0$. Since $\left\{l_{n}\right\}$ is bounded, we have

$$
\lim _{j \rightarrow \infty} \frac{f_{0}^{\left(n_{j}+l_{n_{j}}\right)}}{x^{n_{\jmath}+l_{n_{j}}}} \geq \lim _{j \rightarrow \infty} \frac{W_{n_{\jmath}}}{x^{n_{\jmath}}}\left(\frac{1}{x^{l_{n_{j}}}}\right)>0,
$$

hence $F\left(\Gamma_{0}, 0, x^{-1}\right)=\infty$. By the proof of Lemma (3.5) we note that $L_{0}=x^{-1}$, hence $F\left(\Gamma_{0}, 0, L_{0}\right)=\infty$ and by Theorem $(2.3) \Gamma_{0}$ is positive recurrent.

We consider two examples. The first shows that positive recurrence and equality of $h_{G}$ and $h^{*}$ can happen without $\left\{l_{n}\right\}$ being bounded. The second shows we may have positive recurrence with $h_{G}<h^{*}$. 
EXAMPLE (3.7). Let $\Gamma_{0}\left(\left\{r_{n}\right\},\left\{l_{n}\right\}\right)$ be given by

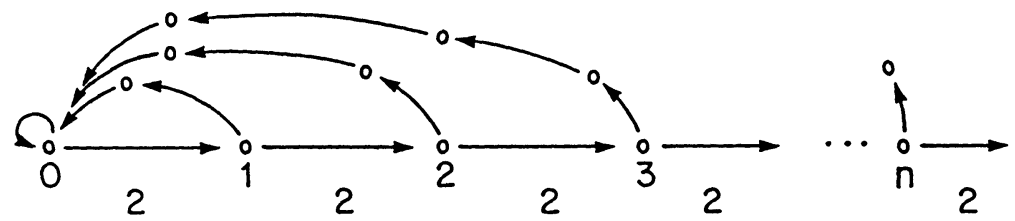

That is $r_{n}=2$ for all $n \geq 1$ and $l_{n}=n+1, n=0,1,2, \ldots \Gamma_{0}$ may be "represented" in a locally finite form by $\Gamma_{0}^{\prime}$ as follows:

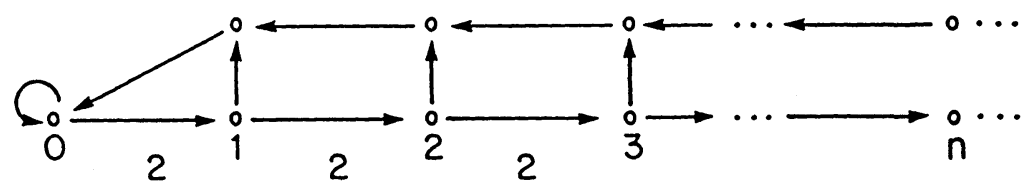

(These two graphs have the same values of $h_{G}$ and $h^{*}$.)

Note that $L_{0}=1 / \sqrt{2}$ and $F\left(\Gamma_{0}, 0, L_{0}\right)=\infty$. Thus, by Theorem (2.3) $\Gamma_{0}$ is positive recurrent. Also $F\left(\Gamma_{0}, 0, \frac{1}{2}\right)=1$, hence $h_{G}\left(\Gamma_{0}\right)=\log 2$. Finally, since $W_{0}=\frac{1}{2}$, then by Lemma (3.2) $h^{*}\left(\Gamma_{0}\right)=\log 2$.

EXAMPLE (3.8). Let $\Gamma_{1}\left(\left\{r_{n}\right\},\left\{l_{n}\right\}\right)$ be the same as the $\Gamma_{0}$ in Example (3.7), except with the loop based at 0 removed. Again by the same argument as in Example (3.7), $\Gamma_{1}$ is positive recurrent. Since $\Gamma_{1}<\Gamma_{0}$, then by Theorem (2.3) $h_{G}\left(\Gamma_{1}\right)<h_{G}\left(\Gamma_{0}\right)=\log 2$. By Lemma (3.2), $h^{*}\left(\Gamma_{1}\right)=\log 2$.

THEOREM (3.9). Given $1<\alpha \leq \beta$, there exists a recurrent uniformly locally finite graph $\Gamma_{0}$ such that $h_{G}\left(\Gamma_{0}\right)=\log \alpha$ and $h^{*}\left(\Gamma_{0}\right)=\log \beta$. If $\alpha=\beta$, then the $\Gamma_{0}$ that we construct is positive recurrent.

Proof. Let $m<\beta \leq m+1$, and choose a sequence $\left\{r_{n}\right\}$ satisfying the conditions of Lemma (3.3) with $\beta$ replacing $x$. We construct the $k$ th partial sum, $S_{k}$, for the loop series of $\Gamma_{0}$ as follows: Let $n_{1}=1$ and set $S_{1}(\alpha) \equiv 1 / \alpha<1$. Let $n_{2}$ be the smallest integer such that

$$
S_{2}(\alpha)=S_{1}(\alpha)+\frac{r_{1}}{\alpha^{n_{2}}}<1 \text {. }
$$

Note that $n_{2} \geq 2$. Assuming that we have $n_{1}<n_{2}<\cdots<n_{k}$, let $n_{k+1}$ be the smallest integer such that

$$
S_{k+1}(\alpha)=S_{k}(\alpha)+\frac{\prod_{i=1}^{k} r_{i}}{a^{n_{k+1}}}<1,
$$

and note that $n_{k+1}>n_{k}$. 
We construct $\Gamma_{0}$ as follows: Let $l_{0}=1$, and $l_{i}=n_{i+1}-i, i=$ $1,2, \ldots$ Then $\Gamma_{0}$ is the graph indexed by the sequences $\left\{r_{n}\right\},\left\{l_{n}\right\}$, and is denoted by $\Gamma_{0}\left(\left\{r_{n}\right\},\left\{l_{n}\right\}\right)$.

Let $S(x)=\lim _{k \rightarrow \infty} S_{k}(x)$. Note that $S(x)$ is the loop series for the graph $\Gamma_{0}\left(\left\{r_{n}\right\},\left\{l_{n}\right\}\right)$, and $S(\alpha)=1$. To show that $h_{G}\left(\Gamma_{0}\right)=\log \alpha$, let $R_{0}$ be such that $h_{G}\left(\Gamma_{0}\right)=\log R_{0}^{-1}$. Thus $F\left(\Gamma_{0}, 0, \alpha^{-1}\right)=1$ and $F\left(\Gamma_{0}, 0, R_{0}\right) \leq 1$. Hence $R_{0} \leq \alpha^{-1}$. If in fact $R_{0}<\alpha^{-1}$, then we must have $F\left(\Gamma_{0}, 0, R_{0}\right)<1$. In this case $\Gamma_{0}$ is transient, hence by Theorem (2.3) $R_{0}=L_{0}<\alpha^{-1}$, contradicting that $L_{0}$ is the radius of convergence of the loop series. Hence $R_{0}=\alpha^{-1}$ and $h_{G}\left(\Gamma_{0}\right)=\log \alpha$. By Lemma (3.2) we have $h^{*}\left(\Gamma_{0}\right)=\log \beta$. Note that if $\alpha<\beta$, then by Lemma (3.5) $\left\{l_{n}\right\}$ is not bounded, hence $\Gamma_{0}$ may be constructed to be a locally finite graph as shown in Example (3.7). Also $\left\{l_{n}\right\}$ is monotone nondecreasing. To show that $\Gamma_{0}$ may be constructed to be uniformly locally finite, we show that if for some $i$ we have $l_{i}=l_{i+T}$, then $(1+T)<\alpha(m+1) / m$. Equivalently, if for some $i$ we have $n_{i+T}=n_{i}+T$, then $(1+T)<\alpha(m+1) / m$.

Let $T$ be the greatest integer such that $n_{1+T}=n_{1}+T=1+T$. Then

$$
S_{1+T}(\alpha)=\frac{1}{\alpha}+\cdots+\frac{\prod_{i=1}^{T} r_{i}}{a^{1+T}} \geq \frac{1}{\alpha}\left(\frac{m}{m+1}\right)+\frac{1}{\alpha}\left(T\left(\frac{m}{m+1}\right)\right) .
$$

Since $S_{1+T}(\alpha)<1$, we have $(1+T)<\alpha(m+1) / m$.

In general, let $k$ be an integer such that $n_{k+1}>n_{k}+1$, and $T$ be the greatest integer such that $n_{k+1+T}=n_{k+1}+T$. Then

$$
\frac{\prod_{i=1}^{k} r_{i}}{\alpha^{n_{k+1}}}+\cdots+\frac{\prod_{i=1}^{k+T} r_{i}}{\alpha^{n_{k+1+T}}}<\frac{\prod_{i=1}^{k} r_{i}}{\alpha^{n_{k+1}-1}}
$$

Hence, $(1+T)<\alpha(m+1) / m$.

Let $d$ be the greatest integer such that $d \leq(m+1) / m$. Then, by construction, $\left\{l_{n}\right\}$ is monotone nondecreasing with the property that it cannot stay constant for more than $d$ consecutive times. Then, by using the same idea as in Example (3.7), it is easy to see that $\Gamma_{0}$ may be constructed to be a uniformly locally finite graph.

Finally, we show that if $\alpha=\beta$ then $\Gamma_{0}$ is positive recurrent. Note that for every $n$ we have

$$
\prod_{i=1}^{n} r_{i}=f_{0}^{\left(n+l_{n}\right)}
$$


Thus

$$
\begin{aligned}
\log L_{0}^{-1} & =\varlimsup_{n} \frac{1}{n+l_{n}} \log f_{0}^{\left(n+l_{n}\right)} \\
& =\frac{1}{1+C} \log W_{0}^{-1}, \quad \text { where } C=\frac{\lim }{n} \frac{l_{n}}{n}
\end{aligned}
$$

Thus we have $L_{0}=W_{0}^{1 /(1+C)}$. Now, for every $n$ we have $l_{n} \geq n / d$, $C=\underline{\lim }_{n}\left(l_{n} / n\right) \geq 1 / d>0$, and $W_{0}<L_{0}$. Since $\alpha=\beta, R_{0}=W_{0}$, we have $R_{0}<L_{0}$ and $\Gamma_{0}$ is positive recurrent.

This result shows that in the Countable-alphabet case, $h_{G}$ and $h^{*}$ can be anything, unlike the finite-alphabet case, where the possible entropies are exactly the Perron numbers (see [7]).

4. Computational examples. In this section we present some computational results of the topological entropy for certain classes of examples of chains. Consider a chain $X$ over $Z \times Z$ with the following transitions: $\left(x_{1}, y_{1}\right) \rightarrow\left(x_{2}, y_{2}\right)$ if either $x_{2}=x_{1} \pm 1$ or $y_{2}=y_{1} \pm 1$. We picture $X$ as follows:

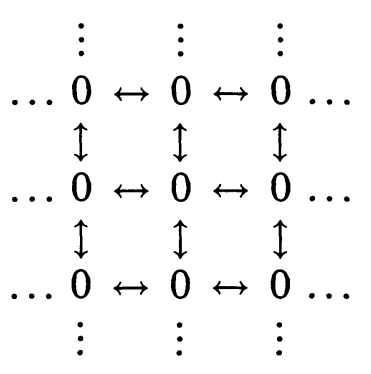

We compute the entropy of some subchains of $X$ whose states are contained in some nice region of $R^{2}$. The following result will be used in the computation.

Let $X$ be a chain with state space $S$, and $f: S \rightarrow C$. Let $f_{\infty}$ be the corresponding map of $X$ generated by $f$ and $Y=f_{\infty}(X)$. For every $n \geq 1$, let $B X(n, s)(B Y(n, c))$ be the set of $n$-blocks in $X(Y)$ starting with $s \in S(c \in C)$; and let $f_{n}$ be the corresponding $n$-block map generated by $f$. For $B \in B Y(n, c)$ let $f_{n}^{-1}(B)=\left\{B^{\prime} \mid B^{\prime} \in B X(n, s)\right.$ and $\left.f_{n}\left(B^{\prime}\right)=B\right\}$.

With these notations we have:

Proposition (4.1). Let $X$ and $Y=f_{\infty}(X)$ be connected chains. If for $i=1,2$ there exists $c_{i} \in C$ and $s_{i} \in f^{-1}\left(c_{i}\right)$ such that

(i) for every $n \geq 1, f_{n}\left(B X\left(n, s_{1}\right)\right)=B Y\left(n, c_{1}\right)$, and 
(ii) $\left\{\# f_{n}^{-1}(B)\right\}$ is bounded as $B$ varies over all blocks in $Y$ starting with $c_{2}$, then $h^{*}(X)=h^{*}(Y)$.

Proof. For $s \in S$ and $c \in C$, let $T_{s}(n)\left(T_{c}^{\prime}(n)\right)$ be the number of blocks in $X(Y)$ starting with $s(c)$. Then

$$
h^{*}(Y)=\varlimsup_{n} \frac{1}{n} \log T_{c_{1}}^{\prime}(n) \leq \varlimsup_{n} \frac{1}{n} \log T_{s_{1}}(n)=h^{*}(X) .
$$

On the other hand, if the bound on $\# f_{n}^{-1}(B)$ is $k$, then we have

$$
h^{*}(X)=\varlimsup_{n} \frac{1}{n} \log T_{S_{2}}(n) \leq \varlimsup_{n} \frac{1}{n} \log \left(k \cdot T_{c_{2}}^{\prime}(n)\right)=h^{*}(Y) .
$$

Remark (4.2). Proposition (4.1) holds for the following two more general cases:

(i) $f$ is an $m$-block map of $X$.

(ii) $X$ and $Y=f_{\infty}(X)$ are both transitive symbolic dynamical systems.

EXAMPLE (4.3). Chains contained in the domain $D(m, n, w)$. Let $D(m, n, w)$ be the domain in $R^{2}$ bounded by two parallel lines of slope $m / n$ with horizontal distance $w$. We assume that one of these lines passes through $(0,0)$. Let $\Gamma(m, n, w)$ be the largest subchain of $X$ contained in $D(m, n, w)$. To compute the $h^{*}$-entropy of $\Gamma(m, n, w)$, we note that it consists of a "fundamental" finite chain $\Gamma_{0}$ which repeats itself periodically in an obvious way giving $\Gamma(m, n, w) . \Gamma_{0}$ may be chosen to include the states $(x, y)$ in $\Gamma(m, n, w)$ such that $y=1,2, \ldots, m$. Let $c$ be the number of states in $\Gamma_{0}$ and consider the following ordering or labelling of these states by $1,2, \ldots, c$ as follows: $\left(x_{1}, y_{1}\right)<\left(x_{2}, y_{2}\right)$ if $y_{1}=y_{2}$ and $x_{1}<x_{2}$ or $y_{1}>y_{2}$. If we repeat this labelling periodically in an obvious way to cover all states of $\Gamma(m, n, w)$, we obtain a one block map, $f$, from the states of $\Gamma(m, n, w)$ to the set $\{1, \ldots, c\}$. The map $f_{\infty}$ generated by $f$ gives a finite chain $\Gamma_{F}(m, n, w)$ over $\{1, \ldots, c\}$ and $f_{\infty}$ satisfies the condition of Proposition (4.1). Hence we have $h^{*}\left(\Gamma(m, n, w)=h^{*}\left(\Gamma_{F}(m, n, w)\right)\right.$.

For example consider $\Gamma(1,2,2)$ :

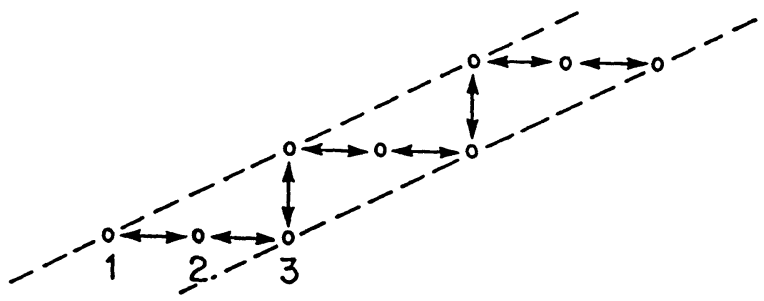


the fundamental chain is given by 0 and may be labelled by 1 , 2 and $3 . \Gamma_{F}(1,2,2)$ is given by

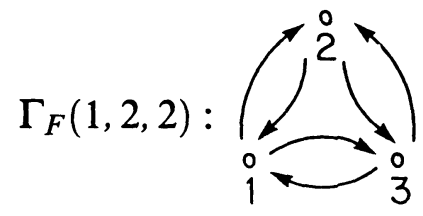

In general, the transition matrix for $\Gamma(1, n, w)$ is given by the $(w+1) \times$ $(w+1)$ matrix $J_{w+1}^{(n)}$, where

$$
J_{w+1}^{(n)}(i j)=1 \quad \text { if }|i-j|=1 \text { or } n, w \geq n>1 .
$$

We note that for $w<n, h^{*}(\Gamma(1, n, w))=0$. Also, for $n=1$, we have

$$
J_{w+1}^{(1)}(i j)=2 \quad \text { for }|i-j|=1 \text {. }
$$

Computation of $h_{G}$ for $\Gamma(m, n, w)$ is generally harder. If we like to compute $h_{G}$ from the definition, then we should be able to identify $h_{G}$ for suitable subchains of $\Gamma(m, n, w)$. This may be easily done in two cases: $\Gamma(1,1, w)$ and the infinite vertical rectangular strip denoted here by $\Gamma(1,0, w)$.

EXAMPLE (4.4). $h_{G}$ for $\Gamma(1,1, w)$. Consider the chain $\Gamma_{l}(1, n)$ given by

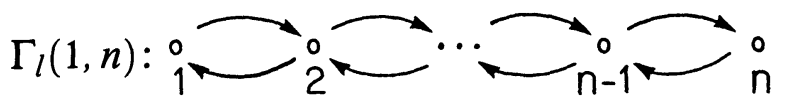

Let $\Gamma_{l}(n \otimes m)$ be the Cartesian product of $\Gamma_{l}(1, n)$ and $\Gamma_{l}(1, m)$. That is, $\Gamma_{l}(n \otimes m)$ is the chain over $\{1, \ldots, n\} \times\{1, \ldots, m\}$ with the following transitions: $\left(x_{1}, y_{1}\right) \rightarrow\left(x_{2}, y_{2}\right)$ if $x_{1} \rightarrow x_{2}$ in $\Gamma_{l}(1, n)$ and $y_{1} \rightarrow y_{2}$ in $\Gamma_{l}(1, m)$. If $T_{n}$ and $T_{m}$ are the transition matrices for $\Gamma_{l}(1, n)$ and $\Gamma_{l}(1, m)$, respectively, then $T_{n \otimes m}=T_{n} \otimes T_{m}$ is the transition matrix for $\Gamma_{l}(n \otimes m)$ (in some order of the state space of $\Gamma_{l}(n \otimes m)$ ), and $h_{G}\left(\Gamma_{l}(n \otimes m)\right)=h_{G}\left(\Gamma_{l}(1, n)\right)+h_{G}\left(\Gamma_{l}(1, m)\right)$. Note that $\Gamma_{l}(n \otimes m)$ is the disjoint union of two chains, and the entropy of $\Gamma_{l}(n \otimes m)$ is the maximum of the entropy of these two chains. Since any finite subgraph of $\Gamma(1,1, w)$ is contained in a component of $\Gamma_{l}((w+1) \otimes m)$ for some integer $m$, we have

$$
h_{G}(\Gamma(1,1, w))=\log \left(2 \times 2 \cos \frac{\pi}{w+2}\right) .
$$

An application of Theorem (2.1) shows that $\Gamma(1,1, w)$ is transient. 
EXAMPLE (4.5). $h_{G}$ for $\Gamma(1,0, w)$ and the Cartesian sum of two chains. The chain $\Gamma(1,0, w)$ is given as

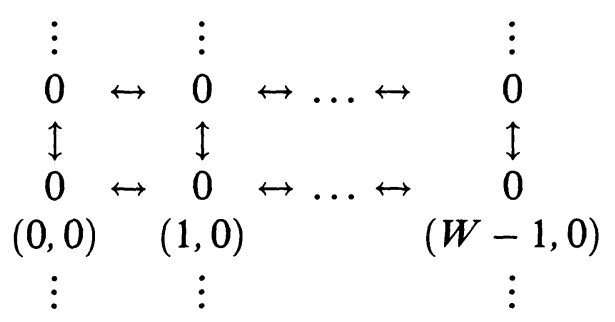

In order to compute $h_{G}(\Gamma(1,0, w))$ we find an easy way to compute the topological entropies of some finite rectangular chains, that is chains of the form

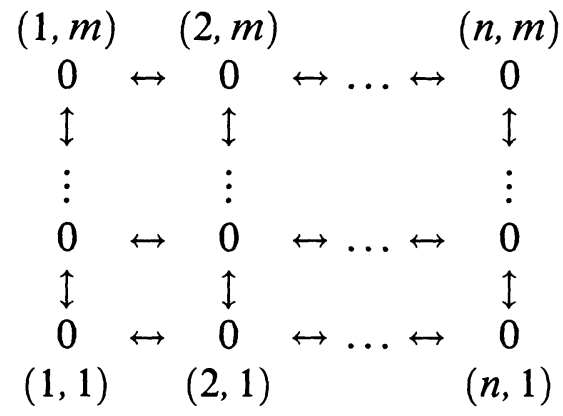

This is done using an idea we introduce as follows. Let $\Gamma_{1}$ and $\Gamma_{2}$ be finite chains with state space $U$ and $V$ respectively. We define the Cartesian sum $\Gamma_{1} \oplus \Gamma_{2}$ to be the chain over $U \times W$ with the following transitions; $\left(u_{1}, v_{1}\right) \rightarrow\left(u_{2}, v_{2}\right)$ if $u_{1} \rightarrow u_{2}$ in $\Gamma_{1}$ and $v_{1}=v_{2}$ or $v_{1} \rightarrow v_{2}$ in $\Gamma_{2}$ and $u_{1}=u_{2}$.

Let $T_{1}$ and $T_{2}$ be the transition matrices for $\Gamma_{1}$ and $\Gamma_{2}$ respectively. Order $U \times V$ according to the following: $\left(u_{1}, v_{1}\right)<\left(u_{2}, v_{2}\right)$ if $v_{1}<v_{2}$ or $v_{1}=v_{2}$ and $u_{1}<u_{2}$. According to this order, the transition matrix $T$ of $\Gamma_{1} \oplus \Gamma_{2}$ is given by $T=T_{1} \otimes I_{m}+I_{n} \otimes T_{2}$. The matrix $T$ satisfies the following two properties: (1) The eigenvalues of $T$ are given by $\alpha+\beta$ where $\alpha$ and $\beta$ are eigenvalues for $T_{1}$ and $T_{2}$ respectively, (2) the eigenvectors of $T$ are given by $X \otimes Y$, where $X$ and $Y$ are eigenvectors for $T_{1}$ and $T_{2}$ respectively.

Now, the finite rectangular chain given above is in fact $\Gamma_{l}(1, n) \oplus$ $\Gamma_{l}(1, m)$ and is denoted here by $\Gamma_{l}(n \oplus m)$. It follows from the previous discussion that

$$
h_{G}\left(\Gamma_{l}(n \oplus m)\right)=\log \left(2 \cos \frac{\pi}{n+1}+2 \cos \frac{\pi}{m+1}\right) .
$$


Direct application of the definition of $h_{G}$ yields:

$$
h_{G}(\Gamma(1,0, w))=\log \left(2+2 \cos \frac{\pi}{w+2}\right) \text {. }
$$

Remark (4.6). Let $D$ be a region in $R^{2}$ such that $D$ contains arbitrarily large rectangles, and let $\Gamma_{D}$ be the largest subchain of $X$ contained in $D$. Then $h_{G}\left(\Gamma_{D}\right)=\log 4$. We also note that for any region $D$, we have $h_{G}\left(\Gamma_{D}\right)=h^{*}\left(\Gamma_{D}\right)$, as indicated in Petersen [9]. We note that the computation for the entropy of $\Gamma_{D}$, where $D$ is a region bounded by two parallel lines of irrational slope, is hard. We feel that the methods introduced here are not going to work in this case. One possible approach may be through studying the continuity properties of $h^{*}$ or $h_{G}$.

Finally, the entropies of the following examples may be computed by first constructing chains which factor onto them. For details see [10].

EXAMPLE (4.7). The sum-bounded systems $X(n, m)$. Consider the closed shift-invariant set

$$
X(n, m)=\left\{\left\{x_{i}\right\}_{-\infty}^{\infty} \mid x_{i}= \pm 1,-n \leq \sum_{l=k}^{K} x_{l} \leq m,\right.
$$

$k$ and $K$ are integers and $n, m>0\}$.

Then for $n, m>0$, the system $X(n, m)$ is sofic with topological entropy

$$
h(X(n, m))=\log \left(2 \cos \frac{\pi}{\min (n, m)+2}\right) .
$$

EXAMPLE (4.8). The sum-bounded-above systems $X(-\infty, m)$. Consider the system $X(-\infty, m)$, where

$$
\begin{aligned}
X(-\infty, m)=\left\{\left\{x_{i}\right\}_{-\infty}^{\infty} \mid x_{i}= \pm 1, \sum_{l=k}^{K} x_{i} \leq m,\right. \\
k \text { and } K \text { are integers and } m>0\} .
\end{aligned}
$$

Then $h(X(-\infty, m))=\log x^{*}$, where $x^{*}$ is the largest root of the equation $(1-x) f_{m+1}(x)+f_{m-1}(x)=0$, and

$$
f_{m}(x)=(-1)^{m} \sum_{k=0}^{[m / 2]}(-1)^{k}\left(\begin{array}{c}
n-k \\
k
\end{array}\right) x^{n-2 k} \text {. }
$$


EXAMPLE (4.9). The sum-bounded run-length-limited systems $X(n, m ; l, k)$. Let $x \in\{-1,1\}^{Z}$ and $B_{r}=x_{1} \cdots x_{r}$ be an $r$-block in $x$. If $x_{1}=\cdots=x_{r}$, then $B_{r}$ is said to be a run of length $r$ of $x . B_{r}$ is a positive (negative) run if $x_{1}=\cdots=x_{r}=1\left(x_{1}=\cdots=x_{r}=-1\right)$. A subblock $B$ of $B_{r}$ is said to be an end run for $B_{r}$ if $B_{r}=x_{1} \cdots x_{i} B$, and $B$ is a run of maximal length, that is $B$ is a run by $x_{i} B$ is not a run.

For $n, m, l$ and $k$ positive integers we define

$$
\begin{aligned}
X(n, m ; l, k)=\left\{x=\left\{x_{i}\right\}_{-\infty}^{\infty} \mid\right. & x_{i}= \pm 1, n \leq \text { the length of a } \\
& \text { positive run in } x \leq m, l \leq \text { the } \\
& \text { length of a negative run in } x \leq k\} .
\end{aligned}
$$

Then, $h(X(n, m ; l, k))=\log x^{*}$, where $x^{*}$ is the largest root of the equation $\sum_{i=n+l}^{m+k} a_{i} / x^{i}=1$ (see Petersen [9]), and the $a_{i}$ 's are given as follows:

Let $M=\min ((k-l)+1,(m-n)+1)$. Then

$$
a_{i}= \begin{cases}i-(n+l)+1 & \text { if } n+l \leq i \leq(n+1)+(M-2), \\ M & \text { if }(n+l)+(M-1) \leq i \leq(m+k)-(M-1), \\ a_{(l+n)+(k+n)-i} & \text { if }(m+k)-(M-2) \leq i \leq m+k, \\ 0 & \text { otherwise. }\end{cases}
$$

Acknowledgment. The author would like to thank Professor Karl Petersen for his invaluable assistance in this research.

\section{REFERENCES}

[1] F. Blanchard, and G. Hansel, Systems codes, Theoretical Computer Science, 44 (1986), 17-49.

[2] F. Blanchard, Systemes dynamiques topologiques associes a des automates recurrents, Z. Wahr. Verw. Geb., 58 (1981), 549-564.

[3] E. E. Dinaburg, An example of the computation of topoligical entropy, Uspehi Mat. Nauk, 23 (1968), 249-250.

[4] B. M. Gurevic, Topological entropy of Enumerable Markov chains, Soviet Math. Dokl., no. 4, 10 (1969), 911-915.

[5] Shift entropy and Markov measures in the path space of a denumerable graph, Soviet Math. Dokl., No. 3, 11 (1970), 744-747.

[6] W. Krieger, On the uniqueness of the equilibrium state, Math. Systems Theory, 8 (1974), 97-104.

[7] D. A. Lind, The entropies of topological Markov shifts and a related class of algebraic integers, Ergodic Theory Dynamical Systems, 4 (1984), 283-300.

[8] K. Petersen, Ergodic Theory, Cambridge University Press, (1983), Cambridge.

[9] Chains, entropy, coding, Ergodic Theory Dynamical Systems, 6 (1986), $415-448$. 
[10] I. A. Salama, Topological entropy and classification of countable chains, Ph.D. dissertation, (1984), Department of Mathematics, University of North Carolina at Chapel Hill.

[11] D. Vere-Jones, Geometric erogodicity in denumerable Markov chains, Quart. J. Math. Oxford, (2), 13 (1962), 7-28.

[12] _ Ergodic properties of nonnegative matrices-I, Pacific J. Math., No. 2, 22 (1967), 361-386.

Received February 16, 1987. This work was partially sponsored by the U. S. Army Research Office Grant No. DAAL03-86-G-0084.

North Carolina Central University

DURHAM, NC 27707 



\section{EDITORS}

\author{
V. S. VARADARAJAN \\ (Managing Editor) \\ University of California \\ Los Angeles, CA 90024 \\ HeRBert Clemens \\ University of Utah \\ Salt Lake City, UT 84112 \\ R. FINN \\ Stanford University \\ Stanford, CA 94305
}

\author{
HERMANN FLASCHKA \\ University of Arizona \\ Tucson, AZ 85721
}

RAMESh A. GANGOLLI University of Washington Seattle, WA 98195

VAUGHAN F. R. JONES University of California

Berkeley, CA 94720
ROBION KIRBY

University of California

Berkeley, CA 94720

C. C. MOORE

University of California

Berkeley, CA 94720

HAROLD STARK

University of California, San Diego

La Jolla, CA 92093

\section{ASSOCIATE EDITORS}
R. ARENS
E. F. BECKENBACH
B. H. NEUMANN
F. WOLF
K. YOSHIDA

(1906-1982)

\section{SUPPORTING INSTITUTIONS}

UNIVERSITY OF ARIZONA
UNIVERSITY OF BRITISH COLUMBIA
CALIFORNIA INSTITUTE OF TECHNOLOGY
UNIVERSITY OF CALIFORNIA
MONTANA STATE UNIVERSITY
UNIVERSITY OF NEVADA, RENO
NEW MEXICO STATE UNIVERSITY
OREGON STATE UNIVERSITY

UNIVERSITY OF ARIZONA

UNIVERSITY OF BRITISH COLUMBIA

UNIVERSITY OF CALIFORNIA

MONTANA STATE UNIVERSITY

NEW MEXICO STATE UNIVERSITY

OREGON STATE UNIVERSITY

\author{
UNIVERSITY OF OREGON \\ UNIVERSITY OF SOUTHERN CALIFORNIA \\ STANFORD UNIVERSITY \\ UNIVERSITY OF HAWAII \\ UNIVERSITY OF TOKYO \\ UNIVERSITY OF UTAH \\ WASHINGTON STATE UNIVERSITY \\ UNIVERSITY OF WASHINGTON
}

The Supporting Institutions listed above contribute to the cost of publication of this Journal, but they are not owners or publishers and have no responsibility for its content or policies.

Mathematical papers intended for publication in the Pacific Journal of Mathematics should be in typed form or offset-reproduced (not dittoed), double spaced with large margins. Please do not use built up fractions in the text of the manuscript. However, you may use them in the displayed equations. Underline Greek letters in red, German in green, and script in blue. The first paragraph must be capable of being used separately as a synopsis of the entire paper. In particular it should contain no bibliographic references. Please propose a heading for the odd numbered pages of less than 35 characters. Manuscripts, in triplicate, may be sent to any one of the editors. Please classify according to the scheme of Math. Reviews, Index to Vol. 39. Supply name and address of author to whom proofs should be sent. All other communications should be addressed to the managing editor, or Elaine Barth, University of California, Los Angeles, California 90024.

There are page-charges associated with articles appearing in the Pacific Journal of Mathematics. These charges are expected to be paid by the author's University, Government Agency or Company. If the author or authors do not have access to such Institutional support these charges are waived. Single authors will receive 50 free reprints; joint authors will receive a total of 100 free reprints. Additional copies may be obtained at cost in multiples of 50 .

The Pacific Journal of Mathematics is issued monthly as of January 1966. Regular subscription rate: $\$ 190.00$ a year (5 Vols., 10 issues). Special rate: $\$ 95.00$ a year to individual members of supporting institutions.

Subscriptions, orders for numbers issued in the last three calendar years, and changes of address should be sent to Pacific Journal of Mathematics, P.O. Box 969, Carmel Valley, CA 93924, U.S.A. Old back numbers obtainable from Kraus Periodicals Co., Route 100, Millwood, NY 10546.

The Pacific Journal of Mathematics at P.O. Box 969, Carmel Valley, CA 93924 (ISSN 0030-8730) publishes 5 volumes per year. Application to mail at Second-class postage rates is pending at Carmel Valley, California, and additional mailing offices. Postmaster: send address changes to Pacific Journal of Mathematics, P.O. Box 969, Carmel Valley, CA 93924.

\section{PUBLISHED BY PACIFIC JOURNAL OF MATHEMATICS, A NON-PROFIT CORPORATION} Copyright (c) 1988 by Pacific Journal of Mathematics 


\section{Pacific Journal of Mathematics}

Vol. 134, No. $2 \quad$ June, 1988

P. D. Allenby and M. Sears, Extension of flows via discontinuous functions

Arthur William Apter and Moti Gitik, Some results on Specker's

problem

Shiu-Yuen Cheng and Johan Tysk, An index characterization of the catenoid and index bounds for minimal surfaces in $\mathbf{R}^{4}$

Mikihiro Hayashi and Mitsuru Nakai, Point separation by bounded analytic functions of a covering Riemann surface

Charles Philip Lanski, Differential identities, Lie ideals, and Posner's theorems

Erich Miersemann, Asymptotic expansion at a corner for the capillary problem

Dietrich W. Paul, Theory of bounded groups and their bounded cohomology

Ibrahim Salama, Topological entropy and recurrence of countable chains

Zbigniew Slodkowski, Pseudoconvex classes of functions. I. Pseudoconcave

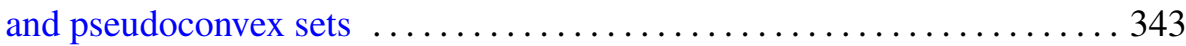

Alfons Van Daele, $K$-theory for graded Banach algebras. II . . . . . . . . . . 377

Edwin Wolf, Functions in $R^{2}(E)$ and points of the fine interior 393 